\title{
Primer trasplante de islotes realizado en Colombia, experiencia fundación Valle del Lili
}

\author{
Gabriel J. Echeverri ${ }^{1}$, Angie N. Sabogal ${ }^{2}$, Luis A. Caicedo ${ }^{1,3}$, Luz. A. Casas ${ }^{4}$, Liliana Mesa, , Johanna \\ Schweineberg ${ }^{5}$, Carlos Durán ${ }^{6}$, Juan G. Posada ${ }^{6}$, Luis G. Arango ${ }^{7}$, Karen M. Feriz ${ }^{4}$, Alfonso J. Holguín ${ }^{8}$, \\ Oscar Gutiérrez ${ }^{9}$, Alejandra Jerez ${ }^{10}$, Jorge I. Villegas ${ }^{1}$, Oscar J. Serrano ${ }^{1}$
}

\begin{abstract}
${ }^{1}$ Médico, especialista en Cirugía de Trasplante de Órganos Abdominales, Fundación Valle del Lili, Escuela de Medicina, Centro para la investigación en cirugía avanzada y trasplantes (CICAT), Universidad ICESI, Cali, Colombia. ${ }^{2}$ Médica Rural, Ingeniera Biomédica, CICAT, Universidad ICESI, Fundación Valle del Lili, Escuela de Medicina, Cali, Colombia.

${ }^{3}$ Director Departamento de Cirugía y Jefe Unidad de Trasplantes, Fundación Valle del Lili, Cali, Colombia.

${ }^{4}$ Médica, especialista en Medicina Interna y Endocrinología, Fundación Valle del Lili, Escuela de Medicina, Universidad ICESI, Cali, Colombia.

${ }^{5}$ Médica, especialista en Medicina Interna y Nefrología, Fundación Valle del Lili, Escuela de Medicina, Universidad ICESI, Cali, Colombia.

${ }^{6}$ Médico, especialista en Medicina Interna y Nefrología, Fundación Valle del Lili, Escuela de Medicina, Universidad ICESI, Cali, Colombia.

${ }^{7}$ Médico, especialista en Medicina Interna y Endocrinología, Fundación Valle del Lili, Escuela de Medicina, Universidad ICESI, Cali, Colombia.

${ }^{8}$ Médico, especialista en Radiología Intervencionista, Fundación Valle del Lili, Escuela de Medicina, Universidad ICESI, Cali, Colombia.

${ }^{9}$ Médico, especialista en Medicina Interna, maestría en Farmacología, Universidad del Valle, Escuela de Medicina, Cali, Colombia.

${ }^{10}$ Médica, candidata a Doctorado en Biomédica, Universidad ICESI, Fundación Valle del Lili, Escuela de Medicina, Cali, Colombia.

Autor para correspondencia:

Dr. Gabriel Jaime Echeverri J. MD,

Cirujano hepatobiliar y de trasplantes, Fundación Valle del Lili Universidad ICESI

Email: gjecheverri@icesi.edu.co
\end{abstract}

\section{Introducción}

$\mathrm{D}$ e acuerdo con las estadísticas suministradas en el 2014 por la Federación Internacional de Diabetes, la prevalencia de pacientes con diabetes está en aumento a nivel mundial, actualmente alrededor de 382 millones de personas sufren de esta patología ${ }^{(1)}$. En relación con la diabetes tipo 1 se estima que corresponde al $5 \%$ de todos los casos diagnosticados de diabetes en adultos ${ }^{(2)}$. Para el caso de Colombia, la incidencia de diabetes tipo 1 es de 3-4 por 100.000 niños menores de 15 años y la prevalencia se estima en $0,07 \%{ }^{(3)}$. Para el municipio de Santiago de Cali, según datos de la oficina de estadística de la Secretaría de Salud Pública, la diabetes (tipo 1 y 2) se encuentra entre las diez primeras causas de muerte en la ciudad, ocupando el octavo lugar en el grupo de hombres y el séptimo entre las mujeres ${ }^{(4)}$. Cabe resaltar que la prevalencia de diabetes para Cali es del 6,76\%, seguida del $5,4 \%$ para el departamento del Valle del Cauca, cifra mucho más alta cuando se compara con la prevalencia nacional que es de $2 \%{ }^{(5)}$.
Específicamente, cuando nos referimos a la diabetes tipo 1, es una patología autoinmune crónica, que se presenta con una predisposición genética específica y en un $0,4 \%$ aparece sin antecedentes familiares ${ }^{(6)}$. Su presentación se hace de forma inesperada, a causa de la destrucción rápidamente progresiva de las células beta pancreáticas, por esta razón requiere la suplencia total de insulina exógena de forma crónica. Lo anterior ocasiona que la enfermedad esté ligada a una carga económica alta para los pacientes, sus familias y el sistema de salud. A nivel global se estima que más del $10 \%$ de la inversión en salud se destina a gastos relacionados directamente con diabetes ${ }^{(7)}$.

En cuanto al tratamiento médico de la diabetes tipo 1, la recomendación actual está basada en la terapia intensiva con insulina exógena, sin embargo, este tratamiento se caracteriza por ser de difícil adherencia, costoso, y por conllevar a un incremento considerable de los eventos de hipoglicemia severa. Estos eventos aumentan en alto grado el riesgo de sufrir deterioro cardiovascular y cognitivo. Por lo tanto, existe una gran necesidad de encontrar mejores alternativas de tratamiento para estos pacientes, que impliquen menores riesgos biológicos y ofrezcan mejor calidad de vida.

Dentro de las opciones terapéuticas se encuentra el trasplante de islotes (TI), procedimiento quirúrgico que en la última década ha tenido un gran desarrollo y se ha considerado recientemente como una opción real y factible para aquellos pacientes con diagnóstico de diabetes que presentan pobre control glicémico, pese a estar bajo regímenes de insulina intensivos ${ }^{(8)}$. Los pacientes con indicaciones para TI (ver tabla 2 de la página 23) típicamente se encuentran bajo el mejor tratamiento médico disponible, sin embargo, persisten con labilidad glicémica y eventos frecuentes de hipoglicemia severa.

Es importante mencionar que dentro de las opciones terapéuticas que se deben considerar para los pacientes con diabetes y pobre control glicémico, se encuentra el trasplante de páncreas (TP) y en el caso de los pacientes con diabetes tipo 1 y enfermedad renal terminal, la mejor opción de control metabólico es el trasplante simultáneo de riñón y páncreas ${ }^{(10)}$. El TP es un tratamiento que ha demostrado beneficios a largo plazo en cuanto a independencia de la insulina; sin embargo, implica una gran morbilidad y mortalidad quirúrgica para los pacientes ${ }^{(11)}$. La tasa de fallas técnicas se encuentra alrededor de 8 a 9\%, la razón más frecuente es trombosis vascular, segui- 
da de infección y pancreatitis ${ }^{(10)}$, por lo que recientemente ha crecido el interés por el TI como una alternativa terapéutica, ya que se realiza una aproximación quirúrgica más segura ${ }^{(11)}$.

El alotrasplante de islotes consiste básicamente en infusiones de islotes aislados de un páncreas cadavérico, trasplantados por vía de la vena porta hacia el hígado (ver figura 2 de la página 25); puede ser realizado como único tratamiento o en asociación con un trasplante renal, en varios casos se realiza en pacientes inmunosuprimidos que han sido llevados previamente a trasplante renal. El obtener un desenlace exitoso posterior al TI significa normoglicemia con reducción o eliminación de la hipoglicemia, por encima de lo que se puede lograr con el gold standard médico terapéutico (múltiples inyecciones de insulina o terapia con bomba de insulina) ${ }^{(12,13)}$.

De acuerdo con el séptimo reporte anual del CITR* (Collaborative Islet Trasplant Registry) sobre la actividad mundial de TI con aloinjerto, se tenía un registro de 571 receptores al año 2010, quienes recibieron 1.072 infusiones de islotes provenientes de 1.187 donantes. Los procedimientos de TI fueron realizados en Norteamérica (66\%) y en Europa y Australia (34\%). Recientemente, se ha reportado un tasa de independencia de la insulina de $50 \%$ a cinco años ${ }^{(14)}$. Datos de centros especializados en este procedimiento reportan que en pacientes que recibieron múltiples y seriadas infusiones de islotes fue posible un $57 \%$ de independencia de la insulina a largo plazo $^{(13)}$. Mediante protocolos innovadores de terapia inmunosupresora ha sido posible obtener altas tasas de independencia a largo plazo, en receptores de islotes provenientes de un donante único, estos protocolos combinan bloqueadores coestimulantes como efalizumab (anticuerpos monoclonales anti-LFA-1) y belatacept o abatacept (inmunoglobulina CTL4), evitando o minimizando el uso de esteroides e inhibidores de calcineurina $\left(\mathrm{CNI}^{*}\right)^{(15,16)}$. Se ha demostrado éxito terapéutico a bajas dosis de CNI. Se reportó que $100 \%$ de los pacientes con TP bajo regímenes terapéuticos que incluyen CNI presentan capacidad secretora normal de las células beta ${ }^{(17)}$, sugiriendo que estos agentes se pueden utilizar e incluso ser necesarios para el éxito de la terapia inmunosupresora en $\mathrm{TI}^{(18)}$. Estos resultados demuestran el gran esfuerzo en investigación invertido en la obtención de mejores protocolos de inmunosupresión.

Se destaca que pos-TI la prevalencia de eventos de hipoglicemia severa disminuye dramáticamente y se determinó que el TI es un factor protector de la tasa de filtración glomerular, con una mayor reducción de las complicaciones microvasculares a largo plazo cuando se compara con los pacientes que reciben tratamiento médico intensivo con insulina ${ }^{(19)}$.

Sin embargo, existe un alto porcentaje de pérdida de islotes; se ha encontrado una relación directa entre la respuesta inflamatoria denominada reacción inflamatoria instantánea mediada por sangre (IBMIR*) y la gran pérdida de islotes que ocurre inmediatamente después de la infusión. Dicha respuesta inflamatoria inicia con la activación del complemento y la cascada de coagulación, que conlleva a formación de coágulos e infiltración de leucocitos en los islotes, comprometiendo así la integridad del injerto, llevando a su destrucción. Este daño inicial y la posterior reacción de rechazo de trasplante ocasionan falla temprana de los islotes y traen como consecuencia periodos cortos de independencia de la insulina ${ }^{(20)}$.

El objetivo de esta publicación es presentar el primer caso de TI realizado en Colombia, que requirió un trabajo multidisciplinario para implementar el proceso de aislamiento de islotes de Langerhans de donante cadavérico, que hizo posible obtener el injerto de islotes que se infundieron al paciente por medio de una intervención quirúrgica realizada por el equipo interdisciplinario de la Unidad de Trasplantes y la Unidad de Endocrinología de la Fundación Valle del Lili, en asociación con la Universidad del Valle y la Universidad de Pittsburgh, en abril de 2009. Consideramos que es importante motivar el desarrollo de terapias de última generación en el país, que permitan ofrecer a los pacientes con diabetes las mejores alternativas disponibles a nivel mundial.

\section{Reporte de caso}

Paciente de sexo masculino de 28 años, procedente de Cali, afrocolombiano, con antecedente de diabetes tipo 1 diagnosticada desde los 11 años. Adicionalmente, hipertensión arterial desde los 17 años, dislipidemia y síndrome convulsivo. Ingresó a hemodiálisis por insuficiencia renal crónica (IRC) a los 23 años hasta el 2008, cuando recibió trasplante renal de donante cadavérico y se inició tratamiento inmunosupresor consistente en tacrolimus $3 \mathrm{mg} /$ día, micofenolato $720 \mathrm{mg} /$ día y prednisolona $5 \mathrm{mg}$ /día. Concomitantemente para el manejo de su diabetes recibía tratamiento con insulina glargina 40 UI/día e insulina lispro 3 UI preprandiales, con pobre control glicémico y múltiples ingresos al servicio de urgencias por hipoglicemias severas con neuroglicopenia, pérdida de la conciencia y síndrome convulsivo secundario.

El páncreas para trasplante fue obtenido de un donante cadavérico, de sexo femenino de 15 años de edad, con un peso de $40 \mathrm{Kg}$, talla $140 \mathrm{~cm}$, IMC de 20,41, cuya causa de muerte cerebral fue hidrocefalia obstructiva. El procesamiento de los islotes de Langerhans a partir de tejido pancreático recolectado el 29 de abril tuvo un tiempo de isquemia en frío de seis horas y se realizó la preservación del tejido con HTK, Custodiol ${ }^{\circledR}$. El aislamiento se realizó en el cuarto limpio de la Universidad del Valle en conjunto con el grupo de investigación médico de la Universidad del Valle y en colaboración con la Universidad de Pittsburgh, siguiendo el protocolo de procesamiento de páncreas descrito por los grupos de trasplante de islotes de Pittsburgh y del Instituto de Investigación de la Diabetes (DRI*). Fue posible obtener un total de 300.000 IE por lo cual la infusión de islotes para el paciente (peso $57 \mathrm{Kg}$, talla $169 \mathrm{~cm}$, IMC 19,96) de acuerdo con su peso fue de $5.263 \mathrm{IE} / \mathrm{Kg}$, con una viabilidad de $70 \%$, islotes agregados mayor del 90\% con recuperación aproximada del $90 \%$ posdigestión. La pureza de los islotes obtenida fue del 30 al 35\%, se procedió a empaquetar en bolsa según protocolo con ciprofloxacina de $20 \mu \mathrm{g} / \mathrm{mL}, 2 \mathrm{mg}$ en 100 
$\mathrm{mL}, 4 \mathrm{mg}$ en $200 \mathrm{~mL} / \mathrm{s}$ en total y heparina de 100 unidades $/ \mathrm{kg}$ en bolo. Se realizó cultivo de los islotes por 12 horas.

El grupo de trasplantes de la Fundación Valle del Lili procedió a llevar al paciente al procedimiento quirúrgico, a quien previamente se le realizó inducción con alemtuzumab $30 \mathrm{mg}$. Se llevó a cabo un abordaje con incisión de minilaparotomía de McBurney en fosa ilíaca derecha, vía vena ileal y vena porta; con ayuda de radiología intervencionista se realizó la infusión de los islotes a goteo lento durante 30 minutos, con supervisión permanente de endocrinología. No se registraron complicaciones durante el procedimiento.

Se estableció un protocolo de seguimiento en el que se realizaron mediciones de glicemia, insulina y péptido $C$ cada 15 minutos hasta completar una hora pos-TI. Para la primera media hora el paciente se encontraba con goteo de dextrosa al $10 \%$ en agua destilada sin insulina, obteniendo los siguientes datos (tabla 1 y figura 1).

Tabla 1. Parámetros registrados posinfusión de islotes; se mantuvo al paciente con infusión de dextrosa al $10 \%$ en agua destilada, sin insulina

\begin{tabular}{l|c|c|c|}
\hline & Posinfusión & $\mathbf{1 5}$ minutos & $\mathbf{3 0}$ minutos \\
\hline Péptido C (ng/mL) & $<0,1$ & 1,53 & 2,45 \\
\hline Insulina (Ul/mL) & 23,4 & 33,5 & 31,5 \\
\hline Glicemia (mg/dL) & 170 & 189 & 185 \\
\hline
\end{tabular}

Tabla 2. Parámetros registrados posinfusión de islotes; se mantuvo al paciente con infusión de dextrosa al $10 \%$ en agua destilada sin insulina

\begin{tabular}{l|c|c}
\hline & $\mathbf{4 5}$ minutos & $\mathbf{6 0}$ minutos \\
\hline Péptido C (ng/mL) & 2,47 & 1,54 \\
\hline Insulina (UI/mL) & 31,1 & 25,4 \\
\hline Glicemia (mg/dL) & 183 & 174 \\
\hline
\end{tabular}

Posteriormente, se realizaron mediciones a los 45 y $60 \mathrm{mi}-$ nutos de los parámetros previamente mencionados con la infusión de dextrosa al 10\% en agua destilada, con el fin de corroborar el adecuado funcionamiento del injerto de islotes. Los datos obtenidos se ven en la tabla 2 .

Como tratamiento inmunosupresor se aumentó la dosis de tacrolimus a $4 \mathrm{mg} /$ día para alcanzar niveles entre 5-7 ng/ $\mathrm{mL}$ en sangre. El paciente fue trasladado a la Unidad de Cuidados Intensivos en donde permaneció durante cinco días. Adicionalmente, se administró piperacilina-tazobactam durante las 48 horas pos-TI, previa toma de hemocultivo y urocultivo; dado que los resultados de estos estudios fueron negativos se suspendió la antibioticoterapia. Posteriormente, el paciente estuvo en hospitalización durante seis días, periodo en el que se ajustaron las dosis de insulina. Se le dio salida al paciente con glicemias entre $91-157 \mathrm{mg} / \mathrm{dL}$ y con requerimientos de insulina lantus 48 UI y lispro 2-6-2 UI preprandiales. Se realizó ecografía Doppler de seguimiento en la circulación portal, en la cual no se encontraron complicaciones.
Figura 1. Datos obtenidos de los análisis sanguíneos realizados posteriormente a la infusión de islotes para insulina, glicemia y péptido $C$ en intervalos de 15 minutos hasta completar una hora de seguimiento

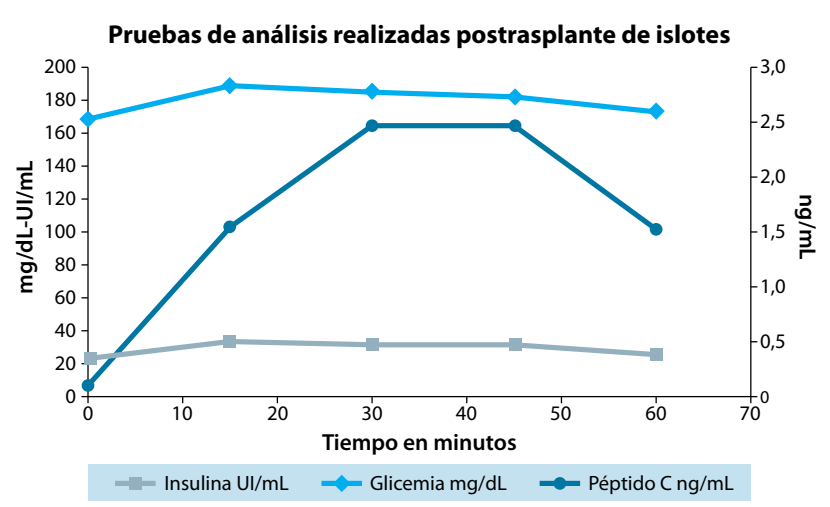

Figura 2. Valores de hemoglobina glicosilada encontrados desde el 2009 hasta el año 2014

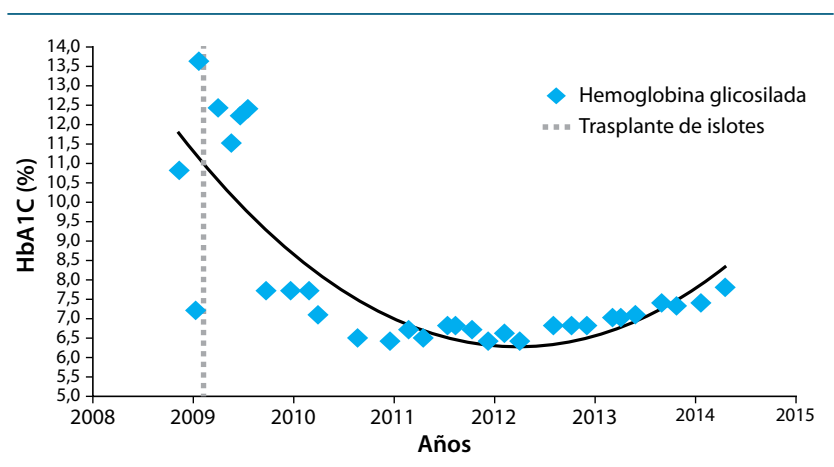

Figura 3. Glicemia basal promedio registrada desde el año 2009 hasta el año 2014

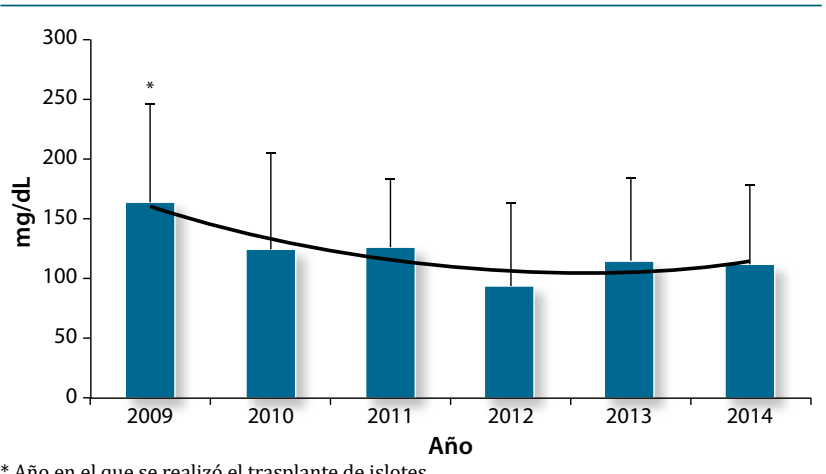

En los controles médicos se registró periódicamente el valor de la hemoglobina glicosilada y glicemia basal (figuras 2 y 3).

Adicionalmente, de acuerdo con los registros clínicos se recopilaron los datos relacionados con el tratamiento de insulina administrado desde el 2008 hasta el 2014. Con el objetivo de poder establecer una mejor relación entre los resultados de análisis sanguíneos obtenidos y el régimen terapéutico al cual fue sometido el paciente (figura 4). 
Figura 4. Unidades de insulina por peso administradas al paciente entre el año 2009 y el 2014

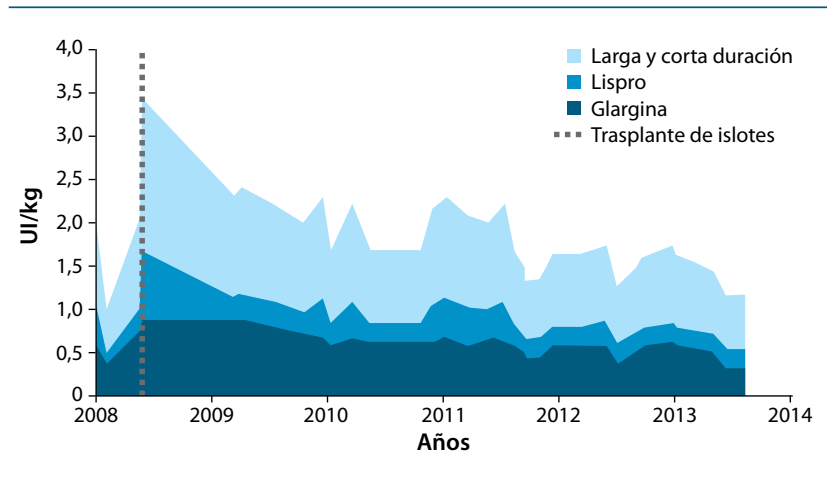

Figura 5. Microfotografía de islotes pancreáticos con tinción de ditizona (aumento 10X)

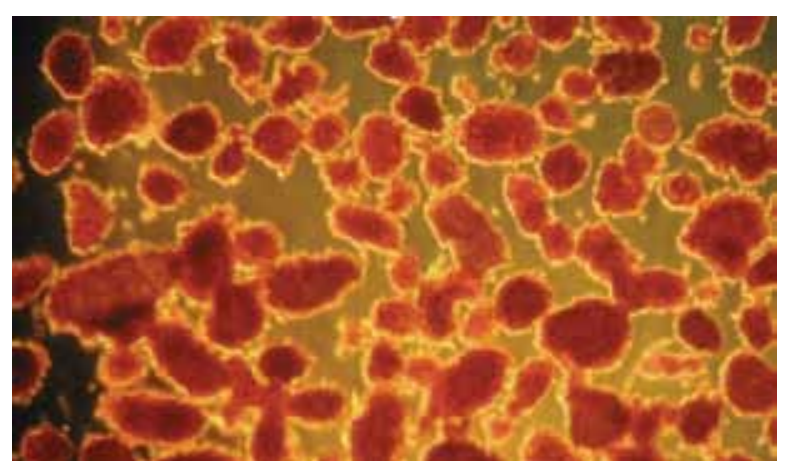

Figura 6. Implantación de islotes pancreáticos en la submucosa gástrica por medio de endoscopia digestiva alta

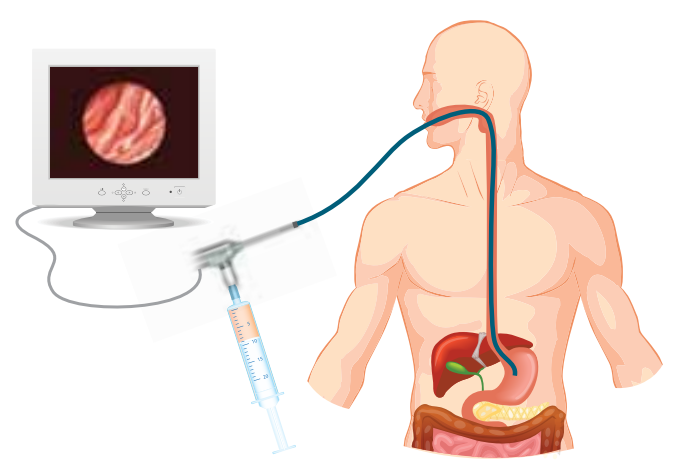

\section{Discusión}

De acuerdo con el seguimiento realizado al paciente, no se registraron complicaciones ni efectos adversos relacionados con el procedimiento quirúrgico. Fue posible evidenciar, después de los 30 minutos de la infusión de los islotes, signos de la respuesta inflamatoria denominada reacción inflamatoria instantánea mediada por sangre (IBMIR) y se registraron niveles de péptido C>0,3 ng/mL y secreción de insulina entre 23,4 y 31,5 UI/mL.

No fue posible obtener un periodo de independencia total de la insulina posterior al TI, este hecho puede estar relacionado con el número de islotes equivalentes infundidos que fue inferior a las recomendaciones de 11.000 a $15.000 \mathrm{IE} / \mathrm{kg}^{(21)}$.
De acuerdo con la literatura, un punto clave es la recepción de páncreas provenientes de múltiples donantes, esto permite la obtención de una masa suficiente para alcanzar los islotes equivalentes requeridos para el paciente ${ }^{(22,23)}$. Recientemente se reportó que los receptores de islotes de un único donante tienen independencia de la insulina y positividad del péptido C más cortas, comparados con pacientes que recibieron dos trasplantes de islotes secuenciales, lo que enfatiza la importancia de la masa de islotes trasplantada para la función del injerto a largo término ${ }^{(24)}$.

Pese a no obtener independencia de la insulina en este paciente, fue posible evidenciar que posterior al TI hubo mejoría sustancial en el control metabólico dado por mejores resultado en los análisis sanguíneos para glicemia basal promedio y hemoglobina glicosilada (figuras 2 y 3). Cabe destacar que, aunque se observan mejores valores de glicemia basal promedio y hemoglobina glicosilada en los años recientes a la realización del tratamiento, los datos para el 2013 son mejores que los registrados previamente al tratamiento, lo que hace suponer que los beneficios metabólicos aún persisten.

Adicionalmente, se registró menor frecuencia de ingreso al servicio de urgencias por año relacionados con eventos de hipoglicemia severa. Así mismo, se disminuyeron complicaciones severas como la neuroglicopenia o los episodios convulsivos.

Con el objetivo de entender los datos relacionados con control metabólico se estudió la administración de unidades de insulina por kilogramo, encontrando una disminución sostenida en el tiempo de los requerimientos de insulina (figura 4). De manera que es posible afirmar que los resultados obtenidos para glicemia basal promedio y hemoglobina glicosilada no se deben a mayores aportes de insulina exógena, por otro lado podrían ser explicados por el TI al que fue sometido el paciente.

Desafortunadamente no se realizaron mediciones de IVGTT, OGTT, test de arginina ni de niveles periódicos de péptido C, para evidenciar la función de los islotes pancreáticos a lo largo del tiempo, lo que no nos permite tener una evidencia clara del funcionamiento de las células beta posterior a la intervención.

Una de las posibles causas de la muerte de las células beta en los TI es el IBMIR ${ }^{(13,25)}$ al exponer los islotes directamente a la sangre y la posibilidad de que no estemos utilizando el sitio adecuado para el trasplante ${ }^{(13,26)}$; por esta razón se han investigado diferentes opciones ${ }^{(27)}$, que ofrezcan un lugar anatómico de máxima implantación del injerto, uso eficaz de la insulina producida y seguridad máxima para el paciente. El trasplante subcutáneo garantiza seguridad y ha sido clínicamente aplicable, otro sitio es el omento que ofrece drenaje de la insulina producida a la vena porta para utilización directa en el hígado, también se encuentra como una opción el trasplante de islotes en la submucosa gástrica por vía endoscópica (figuras 5 y 6), siendo una de las alternativas más prometedoras por la seguridad que ofrece el procedimiento y la posibilidad de realizar varias intervenciones ${ }^{(28)}$. Los esfuerzos futuros están encaminados al diseño de dispositivos que induzcan neovascularización y protejan los islotes del rechazo celular ${ }^{(27)}$. 
Durante el seguimiento periódico realizado desde el 2008 hasta la fecha en consulta externa, se reportó que pos-TI la función renal del paciente ha permanecido estable, pero en los últimos meses, se han evidenciado episodios de hipoglicemia severos con pérdida de conciencia. Por este motivo, el concepto terapéutico emitido por el Grupo de Trasplantes de la Fundación Valle del Lili fue: incluir al paciente en la lista activa para trasplante de páncreas después de riñón; previa discusión del caso de forma amplia y concienzuda es evidente que el efecto que pudo haber representado el TI de islotes, ya no es evidente desde el punto de vista clínico. Además, se descartó llevar nuevamente al paciente a protocolo de infusión de islotes por múltiples razones, entre ellas la consideración administrativa de no tener este tipo de trasplante incluido en el plan obligatorio de salud (POS).

\section{Conclusiones}

El TI constituye una terapia alternativa prometedora para los pacientes con diabetes mellitus tipo 1 . La realización de este procedimiento y su posterior desarrollo en el país constituye un gran logro; de ahí la importancia de la publicación de la experiencia obtenida en la Fundación Valle del Lili, que muestra no sólo las dificultades existentes para el desarrollo de nuevas técnicas quirúrgicas sino el nacimiento de nuevos centros de investigación médica que promueven la implementación de los últimos avances terapéuticos, con el objetivo que facilitar el acceso de los mismos en Colombia.

Es imprescindible destacar lo fundamental del trabajo multidisciplinario desarrollado por el grupo de la Universidad

\section{Referencias}

1. IDF Diabetes Atlas Sixth Edition (www.idf.org/diabetesatlas)

2. National Diabetes Statistics Report 2014. Estimates of Diabetes and Its Burden in the United States (http://www.cdc.gov/diabetes/pubs/statsreport14/national-diabetesreport-web.pdf]

3. Aschner P: Epidemiología de la diabetes en Colombia. Av Diabetol 2010, 26:95-100.

4. Cali AdSd: Cali en cifras 2011. In; 2012.

5. Análisis de situación de Salud Colombia 2013 (http://www.minsalud.gov.co)

6. van Belle TL, Coppieters KT, von Herrath MG: Type 1 diabetes: etiology, immunology, and therapeutic strategies. Physiol Rev 2011, 91(1):79-118.

7. Niclauss N, Morel P, Berney T: Has the Gap Between Pancreas and Islet Transplantation Closed? Transplantation 2014.

8. Pepper AR, Gala-Lopez B, Ziff 0, Shapiro AJ: Current status of clinical islet transplantation. World J Transplant 2013,3(4):48-53.

9. Chaib E, Gama-Rodrigues J, MARJ, Saad WA, Cecconello I: Human islet transplantation - state of the art. Einstein 2005; 3(4):281-28 2005, 3(4):281-285

10. Serrano ÓJ, Villegas JI, Echeverri GJ, Posada JG, Mesa L, Schweineberg J, Durán C, Caicedo LA: Trasplante Simultáneo de riñón y páncreas en pacientes con diabetes mellitus de tipo 1, Clínica Fundación Valle de Lili, Cali, 2001-2013. Rev Colomb Cir, 29.

11. Shapiro AMJ, Ryan EA, Lakey JRT: Clinical islet transplant-state of the art. Transplantation Proceedings 2001, 33(7-8):3502-3503.

12. Ryan EA, Paty BW, Senior PA, Bigam D, Alfadhli E, Kneteman NM, Lakey JR, Shapiro AM: Five-year follow-up after clinical islet transplantation. Diabetes 2005, 54(7):20602069.

13. Vantyghem MC, Marcelli-Tourvieille S, Fermon C, Duhamel A, Raverdy V, Arnalsteen L, Kerr-Conte J, Noel C, Fontaine P, Pattou F: Intraperitoneal insulin infusion versus islet transplantation: comparative study in patients with type 1 diabetes. Transplantation 2009, 87(1):66-71.

14. Bellin MD, Barton FB, Heitman A, Alejandro R, Hering BJ: Potent induction immunotherapy promotes long-term insulin independence after islet transplantation in type 1 diabetes. Am J Transplant 2012, 12(6):1576-1583.

15. Posselt AM, Szot GL, Frassetto LA, Masharani U, Tavakol M, Amin R, McElroy J, Ramos MD, Kerlan RK, Fong L, et al: Islet transplantation in type 1 diabetic patients using calcineurin inhibitor-free immunosuppressive protocols based on T-cell adhesion or costimulation blockade. Transplantation 2010, 90(12):1595-1601.

16. Turgeon NA, Avila JG, Cano JA, Hutchinson JJ, Badell IR, Page AJ, Adams AB, Sears MH, Bowen PH, Kirk AD, et al: Experience with a novel efalizumab-based immunosuppressive regimen to facilitate single donor islet cell transplantation. Am J Transplant 2010, del Valle, la Universidad de Pittsburgh y la Fundación Valle del Lili para conseguir objetivos comunes. En la actualidad no sólo debe existir una confluencia de las áreas del conocimiento sino además de las instituciones que permita el desarrollo e implementación de un manejo óptimo de las patologías.

Es relevante que este es el primer caso reportado de TI realizado en Colombia, por lo que es de gran importancia su publicación, promoviendo de esta forma la construcción de la experiencia y el aprendizaje para la implementación de nuevos tratamientos en el área de trasplantes de células. Razones que son invaluables, cuando se piensa en las múltiples consecuencias positivas que trae el TI con un desenlace exitoso como mejoría de la calidad de vida del paciente. En el futuro es importante incrementar el número de casos, con el fin de obtener más experiencias que contribuyan al desarrollo de este tipo de procedimientos en nuestro centro y poder llegar a conclusiones propias que permitan ofrecer esta terapia al grupo poblacional que se puede beneficiar.

\section{Agradecimientos}

Agradecemos al personal del laboratorio de procesamiento de células de la Universidad del Valle (Fundación INVITRO), al Dr. William Criollo, de la Universidad del Valle, por su asistencia en el proceso de aislamiento, a la Dra. Rita Bottino, de la Universidad de Pittsburgh, por su colaboración en la estandarización del método de aislamiento de islotes pancreáticos, y a la Dra. Anabel Vanín por su asistencia en el procedimiento quirúrgico de trasplante de islotes pancreáticos.

\section{0(9):2082-2091}

17. Rickels MR, Mueller R, Teff KL, Naji A: ?-Cell Secretory Capacity and Demand in Recipients of Islet, Pancreas, and Kidney Transplants. In J Clin Endocrinol Metab. Volume 95; 2010: 1238-1246.(vol 3).

18. Barton FB, Rickels MR, Alejandro R, Hering BJ, Wease S, Naziruddin B, Oberholzer J, Odorico JS, Garfinkel MR, Levy M, et al: Improvement in Outcomes of Clinical Islet Transplantation: 1999-2010. In Diabetes Care. Volume 35; 2012: 1436-1445.(vol 7).

19. Thompson DM, Meloche M, Ao Z, Paty B, Keown P, Shapiro RJ, Ho S, Worsley D, Fung M, Meneilly G, et al: Reduced progression of diabetic microvascular complications with islet cell transplantation compared with intensive medical therapy. Transplantation 2011, 91(3):373-378.

20. Cravedi P, Ruggenenti P, Remuzzi A, Remuzzi G: Chapter 40 - Current Status of Islet Transplantation. In Regenerative Medicine Applications in Organ Transplantation. Edited by Orlando G, Lerut J, Soker S, Stratta RJ. Boston: Academic Press; 2014: 583-598.

21. Corrêa-Giannella ML, Amara ASRd: Pancreatic islet transplantation. Diabetology \& Metabolic Syndrome 2009, 1(9).

22. Shapiro AM: State of the art of clinical islet transplantation and novel protocols of immunosuppression. Curr Diab Rep 2011, 11(5):345-354.

23. van der Windt DJ, Bottino R, Casu A, Campanile N, Cooper DK: Rapid loss of intraportally transplanted islets: an overview of pathophysiology and preventive strategies. Xenotransplantation 2007, 14(4):288-297.

24. Borot S, Niclauss N, Wojtusciszyn A, Brault C, Demuylder-Mischler S, Muller Y, Giovannoni L, Parnaud G, Meier R, Badet L, et al: Impact of the number of infusions on 2-year results of islet-after-kidney transplantation in the GRAGIL network. Transplantation 2011, 92(9):1031-1038.

25. Johansson H, Lukinius A, Moberg L, Lundgren T, Berne C, Foss A, Felldin M, Kallen R, Salmela K, Tibell A, et al: Tissue factor produced by the endocrine cells of the islets of Langerhans is associated with a negative outcome of clinical islet transplantation. Diabetes 2005, 54(6):1755-1762.

26. van der Windt DJ, Marigliano M, He J, Votyakova TV, Echeverri GJ, Ekser B, Ayares D, Lakkis FG, Cooper DK, Trucco M, Bottino R: Early islet damage after direct exposure of pig islets to blood: has humoral immunity been underestimated? Cell Transplant 2012, 21(8):1791-1802.

27. van der Windt DJ, Echeverri GJ, Ijzermans JN, Cooper DK: The choice of anatomical site for islet transplantation. Cell Transplant 2008, 17(9):1005-1014.

28. Echeverri GJ, McGrath K, Bottino R, Hara H, Dons EM, van der Windt DJ, Ekser B, Casu A, Houser S, Ezzelarab M, et al: Endoscopic gastric submucosal transplantation of islets (ENDO-STI): technique and initial results in diabetic pigs. Am J Transplant 2009, $9(11): 2485-2496$ 